\title{
Erratum to: Differences in the metabolic status of healthy adults with and without active brown adipose tissue
}

Qiongyue Zhang · Hongying Ye · Qing Miao · Zhaoyun Zhang · Yi Wang · Xiaoming Zhu · Shuo Zhang · Chuantao Zuo · Zhengwei Zhang · Zhemin Huang · Ruidan Xue · Meifang Zeng · Haiyan Huang • Wanzhu Jin · Qiqun Tang · Yihui Guan · Yiming Li

Published online: 21 March 2014

(C) Springer-Verlag Wien 2014

Erratum to: Wien Klin Wochenschr (2013) 125: 687-695. DOI 10.1007/s00508-013-0431-2
In the published article, the following comment to the authorship is missing:

Q.Z., H.Y., and Q.M. contributed equally to the study.

The online version of the original article can be found under doi:10.1007/s00508-013-431-2

Y. Li $(\bowtie) \cdot$ Q. Zhang $\cdot$ H. Ye · Q. Miao · Z. Zhang · Y. Wang · X. Zhu · S. Zhang $\cdot$ R. Xue $\cdot$ M. Zeng

Division of Endocrinology and Metabolism, Department of Internal Medicine, Huashan Hospital, Fudan University,

12 Wulumuqi Middle Road, Shanghai 200040, China

e-mail: yimingli.fudan@gmail.com

C. Zuo $\cdot$ Z. Zhang $\cdot$ Z. Huang $\cdot$ Y. Guan $(\bowtie)$

PET Center, Division of Nuclear Medicine,

Huashan Hospital, Fudan University,

518 Wuzhong East Road, Shanghai 200235, China

e-mail: guanyihui@hotmail.com

H. Huang · Q. Tang

The Key Laboratory of Molecular Medicine, Ministry of Education and Institute of Biomedical Sciences, Shanghai Medical College,

Fudan University, Shanghai, China

W. Jin

The Key Laboratory of Animal Ecology and Conservation Biology, Institute of Zoology, Chinese Academy of Sciences,

Beijing, China 